\title{
Reciprocal Interactions of the SMA and Cingulate Cortex Sustain Premovement Activity for Voluntary Actions
}

\author{
ㄴ)Vinh T. Nguyen, ${ }^{1,2}$ Michael Breakspear, ${ }^{2,3,4,5}$ and Ross Cunnington ${ }^{1,6}$ \\ ${ }^{1}$ Queensland Brain Institute, The University of Queensland, Brisbane, 4072 Queensland Australia, ${ }^{2}$ QIMR Berghofer Medical Research Institute, Brisbane, \\ Queensland, 4006 Australia, ${ }^{3}$ School of Psychiatry, University of New South Wales, Sydney, New South Wales, 2031 Australia, ${ }^{4}$ The Black Dog Institute, \\ Sydney, New South Wales, 2031 Australia, ${ }^{5}$ The Royal Brisbane and Woman's Hospital, Brisbane, Queensland, 4000 Australia, and ${ }^{6}$ The University of \\ Queensland, School of Psychology, Brisbane, Queensland, 4072 Australia
}

Voluntary action is one of the core functions of the human brain, and is accompanied by the well known readiness potential or Bereitschaftspotential. A network of cortical areas is responsible for the motor preparation process, including the anterior mid-cingulate cortex (aMCC) and the SMA. However, the relationship between activity in these regions during movement preparation and the readiness potential is poorly understood. We examined this relationship by integrating simultaneously acquired EEG and fMRI through computational modeling. We first observed that global field power of premovement neural activity showed a specific correlation with BOLD responses in the aMCC. We then used dynamic causal modeling to infer premovement interactions between these regions and their relationship to the premovement neural activity underlying the readiness potential. These analyses suggest that SMA and aMCC have strong reciprocal connections that act to sustain each other's activity, and that this interaction is mediated during movement preparation according to the readiness potential amplitude, as reflected in global cortical field power. Our study suggests that the reciprocal connections between SMA and aMCC are important to maintain the sustained activity of the readiness potential before movement and lead to a weak system instability at movement onset. We suggest that the effective connectivity of this network underlies its functional role in the preparation of self-generated actions.

Key words: anterior mid-cingulate cortex; multimodal data fusion; readiness potential; simultaneous EEG-fMRI; supplementary motor area; voluntary action

\section{Introduction}

Voluntary actions are planned in the brain before their overt initiation. Understanding the neural activity supporting this planning process is a long-standing challenge in neuroscience. This has been addressed using both EEG and fMRI. Previous EEG studies have reported robust, slowly building neural activity corresponding to the readiness for action (Kornhuber and Deecke, 1964). Activity of this Bereitschaftspotential or "Readiness Potential" (RP) precedes movement onset by 1-2 s, and it is thought to reflect the preparation for voluntary action (Shibasaki and Hallett, 2006). The current consensus is that the RP most likely originates within medial frontal areas (Lang et al., 1991; Praamstra et al., 1996; Ball et al., 1999; Erdler et al., 2000), and subsequently cascades to premotor and primary motor areas (Shibasaki and Hallett, 2006; Haggard, 2008).

fMRI studies have identified a network of cortical areas responsible for the preparation and planning of self-paced move-

Received June 22, 2014; revised 0ct. 23, 2014; accepted 0ct. 25, 2014.

Author contributions: V.T.N., M.B., and R.C. designed research; V.T.N. and R.C. performed research; V.T.N., M.B., and R.C. analyzed data; V.T.N., M.B., and R.C. wrote the paper.

This study was supported by funding from the Australian Research Council (FT0991468, DP110103285).

The authors declare no competing financial interests.

Correspondence should be addressed to Vinh T. Nguyen, QIMR Berghofer Medical Research Institute, Brisbane, Queensland, 4006 Australia. E-mail: vinh.nguyen@qimrberghofer.edu.au.

DOI:10.1523/JNEUROSCI.2571-14.2014

Copyright $\odot 2014$ the authors $\quad 0270-6474 / 14 / 3416397-11 \$ 15.00 / 0$ ments. Most significant and reliable activation has been found in the ACC, the anterior mid-cingulate cortex (aMCC), the SMA, the premotor area (PMA), and the primary motor area (M1; Paus, 2001; Cunnington et al., 2002, 2005; Haggard, 2008; Hoffstaedter et al., 2013). In particular, the SMA is hypothesized to play a crucial role in the higher order organization and preparation of voluntary movements (Yazawa et al., 2000; Cunnington et al., 2003; Shibasaki and Hallett, 2006), while the aMCC is generally thought to play a key role in cognitive aspects of intentional motor control and preparation processes for contextually adaptive actions (Picard and Strick, 1996; Shima and Tanji, 1998). The association between cortical activity recorded by EEG and fMRI of these cortical motor areas is poorly understood, largely due to the difference between spatial and temporal information of the two modalities, as well as their differing neuronal determinants (Logothetis et al., 2001).

Here, we studied the neuronal basis of voluntary movements through the integration of simultaneously acquired EEG and fMRI data. The integration of these data was twofold. First, we assessed trial-by-trial correlations between scalp potentials of the $\mathrm{RP}$ and BOLD signals using EEG-informed fMRI analysis (Eichele et al., 2005). We sought to identify the brain regions from $\mathrm{fMRI}$ associated with greater activity over different stages of the RP during planning and execution of voluntary movement. Second, we applied dynamic causal modeling (DCM; Friston et 
al., 2003) to investigate the effective connectivity of this network and its relation to premovement activity of the RP during the preparation for actions. We primarily focused on a dyadic network consisting of aMCC and SMA, given the effects in our data and the crucial role that these areas are thought to play in movement preparation (Paus et al., 1993; Cunnington et al., 2005; Hoffstaedter et al., 2013, 2014). We aimed to infer effective connectivity between aMCC and SMA in relation to premovement neural activity of the RP during the preparation for self-paced action using model-driven data fusion.

\section{Materials and Methods}

Participants. Twenty right-handed, healthy volunteers (ages 19-29, 7 male, 13 female) took part in the study. All participants disavowed current or prior diagnoses of psychiatric or neurologic conditions or head injury. Participants were recruited from the University of Queensland, and were paid for their participation. Informed consent was obtained from all participants and the study was approved by the Medical Research Ethics Committee of the University of Queensland.

Experimental task. Participants performed a simple task of a brief selfpaced finger sequence movement performed rapidly once every $10-15 \mathrm{~s}$. The movement sequence comprised three rapid alternating button presses, performed on two buttons with the right index/middle index fingers via an MR-compatible button box. A warning message was presented if the time interval between the beginning of a movement and the end of its preceding movement was too short $(<7 \mathrm{~s})$ or too long $(>18 \mathrm{~s})$. Participants were specifically instructed to avoid counting the time between movements in their head, and were given practice at performing the task for several minutes outside the MRI scanner so that they could learn the approximate interval between movements. For the actual (inscanner) experiment, participants received task instruction reminders at the beginning of every run. Each participant performed the task in eight separate blocks (scans) of 6 min each with breaks between blocks as required. This allowed $\sim 30-40$ movements per scan. Participants were required to visually fixate on a cross permanently displayed at the screen center throughout the experiment. An eye-tracking system mounted on the head coil was used to ensure adequate fixation.

Data acquisition. EEG signals were acquired from 64 scalp electrodes positioned according to the international 10-20 system using an MRcompatible EEG system (Brain Products). Reference and ground channels were located anterior and posterior to Fz, respectively. ECG signals were also recorded using a single electrode firmly attached to the participant's back, $\sim 4 \mathrm{~cm}$ left of the spine. Impedance values were kept below $10 \mathrm{k} \Omega$ for EEG channels and $15 \mathrm{k} \Omega$ for the ECG channel. The signals were recorded at a sampling rate of $5000 \mathrm{~Hz}$ and filtered on-line via a low-pass hardware filter at $250 \mathrm{~Hz}$. The sampling intervals on EEG recoding system were synchronized precisely with the MRI clock $(10 \mathrm{MHz})$ via the Brain Products SyncBox device.

fMRI images were acquired on a whole-body 3 T Siemens Trio MRI scanner (Siemens Medical System) equipped with a 12-channel head coil. To minimize head movement, we placed foam padding securely and comfortably around the participant's head without occluding the participant's vision. We used a single-shot gradient-echo EPI sequence with the following parameters: $\mathrm{TR}=1200 \mathrm{~ms}, \mathrm{TE}=35 \mathrm{~ms}, \mathrm{FA}=90^{\circ}$, $\mathrm{FOV}=$ $192 \times 192 \mathrm{~mm}$, pixel bandwidth $2126 \mathrm{~Hz}$, an $84 \times 84$ acquisition matrix, 20 axial slices, and $2.5 \times 2.5 \times 3 \mathrm{~mm}^{3}$ resolution. The axial slices of this EPI sequence were positioned to optimize the coverage of the motor and frontal areas. A total of 300 functional images were acquired for each recording session. The first five images were removed to obtain magnetic equilibrium. A high-resolution T1-weighted structural image covering the entire brain was also collected for each participant with the following acquisition parameters: $\mathrm{TE}=2.32 \mathrm{~ms}, \mathrm{TR}=1900 \mathrm{~ms}, \mathrm{FA}=9^{\circ}, 256 \times$ 256 cubic matrix, and $0.9 \times 0.9 \times 0.9 \mathrm{~mm}^{3}$ voxel size.

EEG analysis. MR gradient artifacts in the EEG signal were removed using the average artifact subtraction method (Allen et al., 2000), in which a template of the MRI artifact was constructed with a sliding average of 31 volumes. Movement parameters, estimated by SPM during the realignment process, were used in building the artifact template. The movement parameters accounted for the jitter of the gradient artifact due to subject movement during acquisition (Moosmann et al., 2009). The artifact-subtracted data were subsequently filtered using a bandpass filter 0.1-45 Hz, and downsampled to $500 \mathrm{~Hz}$.

Following the removal of the gradient artifacts, the EEG data were corrected for ballistocardiogram artifacts using the optimal basis set algorithm implemented in the FMRIB 1.2 toolbox (Niazy et al., 2005). Briefly, EEG signals time locked to heartbeat events ( $\mathrm{R}$ peaks) were extracted and the first three principle components were removed. Next, the EEG data were decomposed using independent component analysis (ICA) implemented in the EEGLAB toolbox (Delorme and Makeig, 2004). The ICA decomposition of our EEG data did not consistently generate ERP-related components for all participants, that is, components showing a movement-locked response and having a topographic distribution of activity over the central channels. Hence, ICA was used for artifact removal rather than extracting RP amplitudes in single trials as used by previous studies (Debener et al., 2005; Eichele et al., 2005). Here, ICA components representing residuals of ballistocardiogram and eye-movement artifacts were identified and removed during the backprojection procedure.

The RP is a spatially broad potential maximal at the vertex, evident across most electrodes on the scalp, and usually evident in the average across all channels. It is therefore standard to calculate RP references to linked mastoids rather than using an average reference. In the present study, the signals were re-referenced to the average signals of TP9 and TP10 as an approximation of the linked mastoids to capture the slow negative slope of the RP. Re-referenced data were segmented into epochs from -2000 to $500 \mathrm{~ms}$ relative to the onset of the first button-press event for each movement, and corrected for a baseline between -2000 and $-1500 \mathrm{~ms}$. Trials were rejected if amplitudes of any channel at any data point exceeded a threshold of $\pm 100 \mu \mathrm{V}$. This exclusion criterion minimizes the impact of residual artifacts directly or indirectly related to head motions induced by the pressing of the response button, such that the integration analysis with fMRI signals (see detail below) was not confounded by the head-motion artifacts (Jansen et al., 2012; Duncan and Northoff, 2013). On average, $16 \%$ of trials were rejected, and $183 \pm 17$ valid trials met the thresholding criteria across all participants.

As the RP is usually maximal over the central channels, we focused our analysis on signals of the left (FC3 and C3), midline (FCz and Cz), and right central and frontocentral channels (FC4 and $\mathrm{C} 4$ ). The lateralized readiness potential (LRP) was computed as the difference between the left (FC3 and C3) and the right central channels (FC4 and C4). The global field power (GFP) was calculated as the square root of the mean of the squared potentials at each electrode across the whole scalp (Lehmann and Skrandies, 1980). To examine the temporal specificity of the BOLD correlates of the readiness potential, we divided the EEG data into four consecutive and nonoverlapping time bins of $250 \mathrm{~ms}$ window between -1000 and $0 \mathrm{~ms}$ before movement. The RP and GFP potentials were averaged across each time window and compared against the baseline activity (zero mean) using single-sample $t$ tests (two-tailed with Bonferroni corrections for the number of time windows and electrodes).

Single-trial EEG estimation. We estimated single-trial GFP, RP, and LRP responses needed for the integration analysis with fMRI data. Here, single-trial GFP responses were computed as the mean potential of each time window. We obtained one value per time window for each trial, which denotes the trial-to-trial variation of GFP during movement preparation.

For RP, single-trial potential amplitudes were estimated from left (FC3/C3), midline (FCz/Cz), and right central channels (FC4/C4). Due to the low SNR of data from single channels (compared with the GFP), we applied an autoregressive with exogenous (ARX) method to better estimate the slow-wave potentials for each trial in these frontocentral channels (Cerutti et al., 1987; Nguyen et al., 2013). The ARX method represents the epoch-specific data as the sum of spontaneous activity, the event-related response (the RP), and noise. The spontaneous activity is modeled as the output of an autoregressive model driven by a whitenoise process. The event-related response is modeled as the output of a moving-average filter driven by the average RP response. In other words, estimates of RP responses in each trial were derived from the average RP 
by an ARX filter that best fits the data. The ARX-filtered data were averaged across data points for each of the $250 \mathrm{~ms}$ time windows before movement to derive the trial-to-trial variation of the RP response for each of the frontocentral regions over each $250 \mathrm{~ms}$ time window.

Finally, single-trial potentials of the LRP were estimated as the difference of the single-trial estimates between the left (FC3/C3) and right frontocentral regions (FC4/C4). As the LRP reflects neural activity closer in time to the execution of movements, with onset $\sim 500 \mathrm{~ms}$ before movement, we only extracted the single-trial LRP responses over the time windows from -500 to $-250 \mathrm{~ms}$ and -250 to $0 \mathrm{~ms}$ before movement onset.

To assess the reliability of our single-trial estimation methods to identify trials with relatively higher or lower amplitudes, we performed a median split on single-trial amplitude measures and recomputed ERPs by averaging trials with high amplitudes compared with low amplitudes. As single-trial amplitudes were estimated independently from consecutive time windows, this also allowed us to examine how specific the highand low-amplitude differences were to the time window in which they were estimated. Hence, this analysis allowed us to assess the reliability of our single-trial estimation method to identify trials with high and low amplitudes in the relevant time windows.

Furthermore, to determine the degree to which premovement GFP activity and RP amplitudes reflect the same underlying process, we examined the correlation between GFP and RP both across the single-trial estimates and across participants. For single-trial analysis, we examined correlations between trial-by-trial GFP amplitudes and RP single-trial amplitude estimates from each of the time windows in the left-central (FC3 and C3), midline-central (FCz and Cz), and right-central channels (FC4 and C4). Across participants, we examined the correlation between mean GFP activity and mean RP amplitude for each participant over grouped frontocentral channels (FC and C row channels) for each time window. We also examined the same correlations in grouped prefrontal channels (AF and Fp rows) and posterior channels ( $\mathrm{P}$ and $\mathrm{PO}$ rows) to compare relationships between GFP activity and RP amplitudes over regions not typically associated with the generation of the RP.

$f M R I$ analysis. Functional images were preprocessed and analyzed using the Statistical Parametric Mapping software (SPM8; Welcome Department of Imaging Neuroscience, Institute of Neurology, London) implemented in MATLAB. The functional images were first realigned temporally by the slice-timing correction procedure, and then spatially realigned to correct for head motion using a six-parameter rigid body spatial transformation. The structural T1 image was coregistered to the mean functional image obtained during the realignment procedure and the coregistered $\mathrm{T} 1$ image was spatially normalized to the MNI template using the Segment procedure of SPM8. The functional images were transformed to the MNI space using the parameters obtained from the transformation of the T1 image, then resliced to a voxel size of $2 \times 2 \times 2$ $\mathrm{mm}^{3}$, and spatially smoothed with a $5 \mathrm{~mm}$ FWHM Gaussian kernel. The T1 image was also resliced to a resolution of $0.5 \times 0.5 \times 0.5 \mathrm{~mm}^{3}$ for display purposes.

We applied an event-related GLM to examine responses related to the planning and execution of voluntary movements. A regressor modeling the onset of the first button-press event for each movement was constructed through the convolution of the event onsets with the canonical HRF. Invalid events, representing button presses that were performed either too early or too late, were modeled as error events in a separate regressor. Head-motion parameters were included in the design matrix as covariates of no interest. The data were filtered using a high-pass filter with a cutoff at $120 \mathrm{~s}$, and an autoregressive model [AR(1)] was applied to correct for temporal autocorrelation in the data.

For each participant, brain activity corresponding to voluntary actions was defined by a $t$-contrast comparing "Movement $>$ Rest," with Rest as an implicit baseline in the GLM design matrix. These contrast images for each individual participant were then entered into a second-level random-effects analysis to examine group-level activation using a singlesample $t$ test. Activity corresponding to the contrast of movement versus resting was identified using a cluster-level threshold $P_{\text {FWE }}<0.05$, corrected for multiple comparisons, with clusters identified using a voxellevel threshold $P_{\text {uncorrected }}<0.00005$. This more stringent height threshold was applied, rather than a more commonly used threshold (e.g., $P_{\text {uncorrected }}<0.001$ ), to better identify the locations of activation peaks in motor-related brain areas. Our study comprised $\sim 200$ trials per participant, which was necessary for the EEG measures, but provided extremely high statistical power for the fMRI analysis. We therefore adjusted the cluster-forming height threshold used for the fMRI analysis accordingly. Anatomical labels for significant activation clusters were identified using the Anatomy toolbox (Eickhoff et al., 2005).

EEG-fMRI integration analysis. We sought to determine which brain regions showed activity correlating with trial-to-trial fluctuations of EEG activity over the different time periods before movement using EEGinformed fMRI analysis (Debener et al., 2005; Eichele et al., 2005; Nguyen and Cunnington, 2014). At the single-subject level, the single-trial measures derived from the EEG were convolved with the canonical HRF and orthogonalized with respect to the standard HRF regressor for event onsets. The orthogonalization procedure essentially mean centered the EEG measures but still maintained their trial-by-trial fluctuation. Hence, these EEG-informed regressors represented parametric modulation of BOLD responses according to the level of premovement EEG activity in each of the different time windows from which the single-trial estimates were derived.

In summary, the design matrices for EEG-informed fMRI analyses included: (1) the standard HRF regressor modeling expected responses to movement events; (2) a parametric-modulator regressor modeling the trial-to-trial variability of the EEG response, representing the correlation between EEG activity and BOLD responses at a trial-by-trial level; (3) a regressor for invalid movements; and (4) regressors of realignment parameters as covariates of no interest. Since the time windows were not linearly orthogonal, the EEG-informed fMRI analysis was performed independently for single-trial EEG measures (i.e., RP, GFP, and LRP) of each time window. In total, we implemented 18 analyses including 12 models for the RP ( 3 channels $\times 4$ time windows), 4 models for the GFP (four time windows), and 2 models for the LRP (two time windows). For group analysis, the parameter estimates for the EEG-related regressors, modeling correlations of trial-by-trial EEG activity with BOLD responses, were entered into second-level random-effects analysis and compared against the zero mean (no correlation) using single-sample $t$ tests. Significant correlations were defined using a cluster-level threshold of $P_{\mathrm{FWE}}<0.05$, with clusters defined by a voxel-level threshold $P_{\text {uncorrected }}<0.001$.

To ensure that results of the EEG-fMRI integration analysis were not influenced by possible differences in overall EEG amplitudes betweensubjects, we additionally performed the EEG-fMRI integration analysis using normalized values for the EEG-derived regressors. Here, singletrial EEG estimates were $z$-transformed before they were entered to the GLM as parametric modulators. The $z$-transformation adjusted not only the mean but also the variance of the single-trial measures, hence removing between-subject differences. Notably, this normalization of values for the EEG-fMRI integration analysis had almost no influence on the results, with both the extent and location of activation almost identical between the two analyses. The results we report below are from the analysis conducted with the single-trial EEG estimates mean centered for orthogonalization but without further normalization.

Dynamic causal modeling of fMRI data. While the EEG-informed fMRI analysis provided specific timing information about the BOLD correlates of movement-related activity, it did not allow interference of causal interactions between active brain regions. The analysis of effective connectivity, on the other hand, allows one to infer interactions between brain regions underlying effects of interest (Friston et al., 2003). In this study, we used DCM to investigate effective connectivity between the core brain regions responsible for the preparation of voluntary actions. Crucially, we incorporated EEG-derived measures in our DCM models to examine how the interactions between these brain regions were modulated when premovement brain activity was high compared with low for each trial. Our analysis was performed using DCM12 implemented in the SPM12b software.

The first step of the DCM analysis was to extract time courses from ROIs. Using results of the group-level GLM analysis, ROIs were extracted from key areas of the motor system involved in the generation of self- 
paced actions. Specifically, from our results (see below), our brain network for DCM analysis comprised regions in the aMCC, SMA, and M1. These regions were identified within each individual using a combination of anatomical and functional criteria (Stephan et al., 2007). The coordinates of the peak (group-level) activation in SMA were defined as the maximum peak lying on the mesial surface within the interhemispheric fissure and belonging to Brodmann's area 6 (BA6), according to the AAL atlas (Tzourio-Mazoyer et al., 2002). Similarly, peak activation in the cingulate cortex was defined as the maximum peak within the interhemispheric fissure in BA32 according to the AAL atlas. In our group analysis, this cingulate peak was located in the anterior portion of the aMCC. Individual ROIs for each participant were then defined as the peak in their single-subject analysis within $5 \mathrm{~mm}$ proximity to the group coordinates. The ROI time series from each of these regions were then extracted from the spheres of $5 \mathrm{~mm}$ radius around these subject-specific peak coordinates. The ROI time series data were adjusted for effects of no interest by regressing out the motion parameters and error trials. Hence, the extracted time series included effects of interest representing BOLD responses in our data associated with the preparation and execution of movement.

DCM is a hypothesis-driven approach and therefore requires specification of a model space that defines specific patterns of interactions between brain regions (Friston et al., 2003). The construction of our model space was tightly constrained to model-specific effects observed in our data, including the observed task effects (from the task-related fMRI analysis) and EEG-BOLD correlation effects (from the EEG-informed fMRI analysis). To examine how connections between brain regions were modulated according to premovement activity from EEG measures, parameters derived from the single-trial EEG responses were entered into our DCM models as contextual (bilinear) modulators. Specifically, we classified trials according to a median split of high versus low EEG responses and entered these as modulators on specific connections between brain regions (nodes in our DCM models) to model the possible causes of the EEG-BOLD correlations observed in specific brain regions. Crucially, we only added contextual modulators that were consistent with the effects observed in our data, thereby constraining our model space only to those models that could explain causes of the actual effects observed. For example, our analysis found a positive correlation between GFP activity and BOLD responses in the aMCC (see Results). Trials of high-GFP responses were therefore added as contextual modulators on those connections that could explain the positive correlation with BOLD responses in the aMCC node (see Results for more detail). We have previously shown this approach to significantly improve overall DCM model performance (Nguyen et al., 2013).

We used variational Bayes to perform model inversion and model comparison (Friston et al., 2003). In this framework, the likelihood of the data given the model is maximized using an iterative maximizationexpectation procedure. Posterior model likelihood (evidence vs complexity) was then derived using a Bayesian framework based on the Free Energy, which is an approximation to the model likelihood (Friston, 2010). For model comparison at the group level, we applied Bayesian model selection (BMS) with random-effects analysis to examine the relative performance of all models (Penny, 2012).

\section{Results \\ ERP results}

As expected, the grand average RP showed a prolonged and increasing negativity preceding movement onset, beginning $\sim 1.5 \mathrm{~s}$ before movement (Fig. 1A). Potentials were maximal over central midline electrodes during early stages, but were increasingly lateralized within $500 \mathrm{~ms}$ of movement onset (Fig. 1C). Statistical comparisons across the four time windows showed that RP amplitudes were significantly greater than baseline from $750 \mathrm{~ms}$ before movement in midline $(\mathrm{FCz} / \mathrm{Cz})$ and left $(\mathrm{FC} 3 / \mathrm{C} 3)$ channels, and from $500 \mathrm{~ms}$ before movement in right (FC4/C4) channels (Fig. $1 B$; all $p<0.004$ ). Analysis of LRPs showed that amplitudes over the left channels were significantly greater than over the right channels only in the last time period, from $250 \mathrm{~ms}$ before movement (Fig. $1 B ; p<0.001$ ). Analysis of the GFP showed significantly greater activity than baseline in all four time windows, from $1000 \mathrm{~ms}$ before movement $(p<0.003)$. Overall, these results indicate a significant increase in scalp potentials beginning as early as $1 \mathrm{~s}$ before movement onset and showing significant lateralization to the left hemisphere within $250 \mathrm{~ms}$ before movement.

To assess the reliability of the single-trial estimates to identify trials with high- and low-amplitude responses in the relevant time windows, we divided trials according to high versus low EEG responses based on the single-trial measures of the GFP, RP, and LRP, and recalculated the average ERPs for the high- and lowresponse trials (Fig. 2). These results clearly show that our singletrial estimation could indeed separate trials with high versus low amplitudes over the identified time windows. This confirms the reliability of the single-trial estimates of premovement activity, and suggests that the EEG-informed fMRI analysis should allow investigation of the BOLD correlations with the premovement activity from these EEG measures at specific times before movement onset. Importantly, the average GFP plots for high- and low-response trials (Fig. 2, bottom row) were progressively different in the consecutive time windows over which GFP amplitudes were estimated. The temporal specificity of the single-trial RP and LRP estimates, however, were less clear. Although we found clear differences in the average ERP between the high- and low-RP trials, the whole waveforms appear higher for trials having large RP estimates, independent of the time window used to estimate the amplitudes. Hence, the trial-by-trial variation of the RP and LRP activity would be less likely to provide temporally specific correlation with BOLD responses when these single-trial measures were entered into the EEG-informed fMRI analysis.

We also examined the correlation between GFP activity and RP amplitudes, to determine the relationship between GFP and RP over the premovement period (Fig. 1D). In single-trial analysis, we found no significant correlation between trial-by-trial GFP amplitudes and single-trial estimates of RP amplitudes. We suggest that this reflects the difficulty in obtaining reliable singletrial estimates of premovement brain activity from the RP, due to the very low signal-to-noise ratio of the RP. Assessing correlation across participants, however, we found strong and highly significant correlations between mean GFP and mean RP amplitudes over frontocentral channels from the second time bin onward (from $-750 \mathrm{~ms}$ before movement), with the strongest correlation in the last time bin immediately before movement onset ( $r=$ $-0.75, p<0.0001)$. The prefrontal channels showed much weaker correlation, but still significant effects in the last two time bins $(r=-0.56, p<0.01)$. Posterior channels showed no significant correlations $(p>0.05)$. These results suggest that the GFP captures highly similar activity to RP amplitudes over the frontocentral channels, and that contributions of other prefrontal or posterior brain regions to GFP activity over this premovement period are only minor.

\section{Whole-brain fMRI results}

Whole-brain fMRI analysis revealed key regions of the motor system that were active in response to the planning and execution of self-paced actions. Overall, as expected for right-hand movements, activation was stronger and spatially more extensive in motor areas of the left hemisphere than the right (Fig. 3). Significant activation clusters were localized to the mid-cingulate cortex (MNI: $x=2, y=10, z=38, t=6.14$ ), the supplementary motor area (MNI: $x=-2, y=-6, z=54, t=10.74$ ), the left premotor area (MNI: $x=-30, y=-16, z=64, t=11.12$ ), the 
A
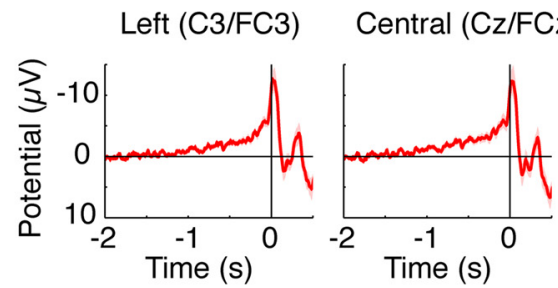

B
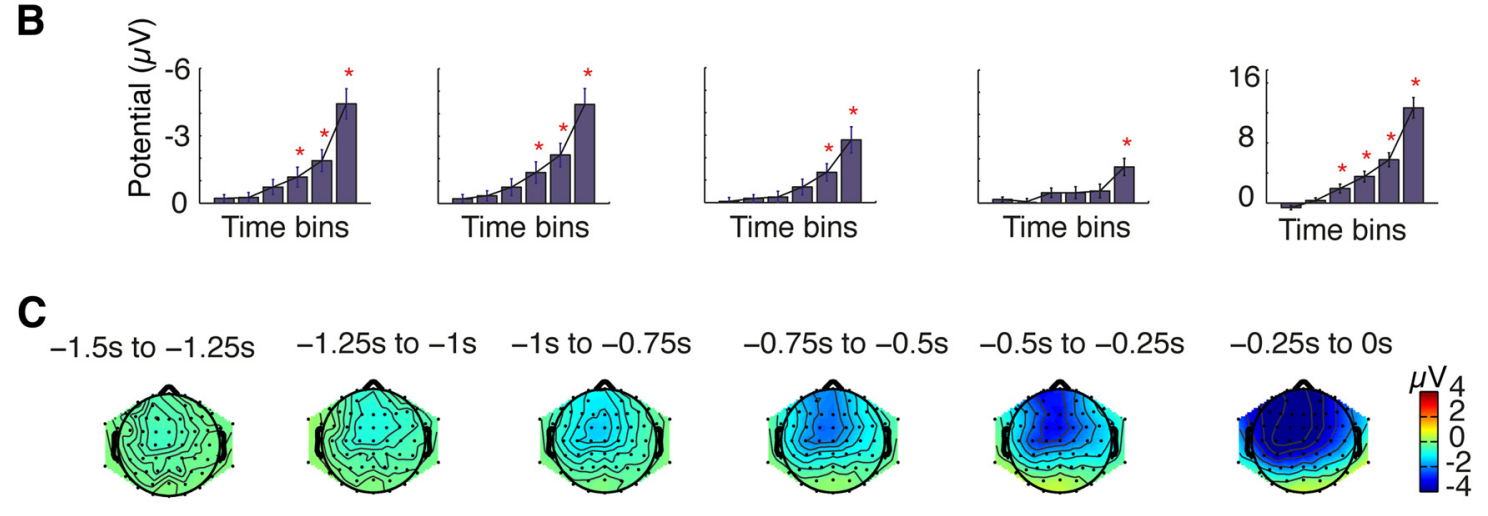

D
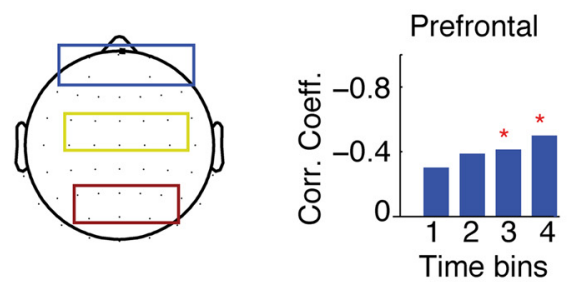
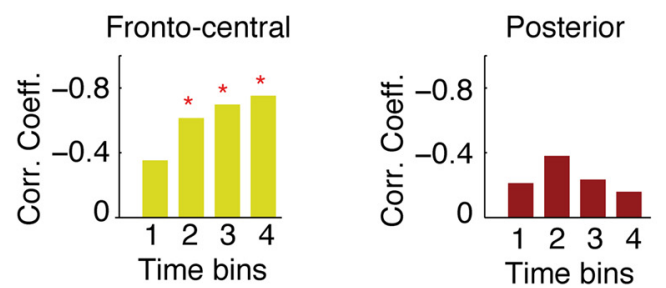

Figure 1. Results of the conventional EEG analysis. $\boldsymbol{A}$, Grand average waveforms of the central EEG channels (left, central, and right), the LRPs, and the GFPs are depicted, showing a typical morphology of increasing negative potential shift in premovement activity. $\boldsymbol{B}$, Waveforms were divided into six time windows of 250 ms intervals. The potentials of the time windows were compared against the zero mean baseline, with significant differences from baseline indicated by red asterisks. $C$, Topographic maps of these time bins showed a symmetrical distribution of the RP potentials during the early component, with activity becoming stronger on the left hemisphere contralateral to the movement side immediately before action. $D$, Correlation between GFP activity and mean RP amplitudes across subjects for the four time bins from - 1000 to 0 ms before movement over prefrontal channels (blue), frontocentral channels (yellow), and posterior channels (red). Strong and highly significant correlations were found between GFP amplitudes and mean RP amplitudes over frontocentral channels, with weaker correlation over prefrontal channels, and no significant correlation over posterior channels.

left primary motor area (MNI: $x=-38, y=-24, z=50, t=$ 12.03), and the left dorsolateral prefrontal cortex (MNI: $x=-60$, $y=8, z=20, t=10.84$ ). The only right hemisphere region with significant activation was in the medial frontal cortex (MNI: $x=$ $34, y=50, z=24, t=14.23$ ).

\section{EEG-fMRI integration analysis}

The EEG-informed fMRI analysis revealed a positive correlation between trial-by-trial variation of premovement GFP activity and a cluster of activation in the cingulate cortex (Fig. 4). Over the time window from 750 to $500 \mathrm{~ms}$ preceding movement, clusters in the ACC and mid-cingulate cortex showed positive correlation with the GFP activity. The peak of this cingulate activation was located in the anterior portion of the aMCC (MNI: $x=-8, y=$ $14, z=41, t=5.26$ ) corresponding closely with the aMCC coordinates reported in previous studies (Hoffstaedter et al., 2013, 2014). Similarly, the premovement GFP activity over the time window from 500 to $250 \mathrm{~ms}$ was correlated with activation in the same regions of the ACC and mid-cingulate cortex, with the peak again localized to the aMCC (MNI: $x=4, y=12, z=39, t=5.08$. No significant correlation was identified in any of the other time windows, and no regions showed significant correlation of BOLD responses with trial-by-trial variation in RP or LRP amplitudes over any of the time windows, even using a less stringent voxel- level threshold $p_{\text {uncorrected }}<0.005$. Furthermore, no regions showed significant negative correlations between BOLD responses and trial-by-trial fluctuations of premovement GFP or RP/LRP amplitudes over any of the time windows.

To refine the temporal precision of the GFP-BOLD correlation results, we performed similar EEG-informed fMRI analyses, but with shorter $100 \mathrm{~ms}$ time windows. These analyses revealed significant correlations between GFP and BOLD responses in the three consecutive time windows from 700 to $400 \mathrm{~ms}$ before movement onset, again with significant voxels in aMCC and ACC (cluster-level $p_{\mathrm{FWE}}<0.05$, corrected for whole-brain analysis). These results show that BOLD activation responses in the aMCC positively correlate with the amplitude of neural activity in the GFP over the period from 700 to $400 \mathrm{~ms}$ before movement. It is worth noting that we found robust activation in the aMCC corresponding to task effects as well as trial-by-trial variation of GFP activity. This argues that correlations we find in the aMCC are associated with premovement preparation and are not a confound of spurious trial-by-trial variation unrelated to the task. Hence, overall we find significant activation in the mid-cingulate cortex associated with voluntary action and, crucially, the level of this activation correlates significantly with premovement GFP activity such that trials in which premovement neural activity is 

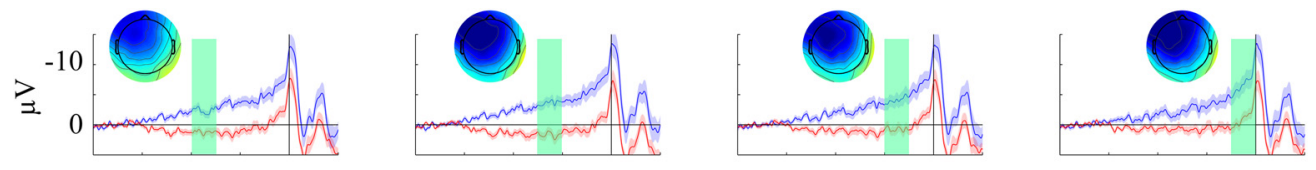

$\mathrm{FC} 3 / \mathrm{C} 3$
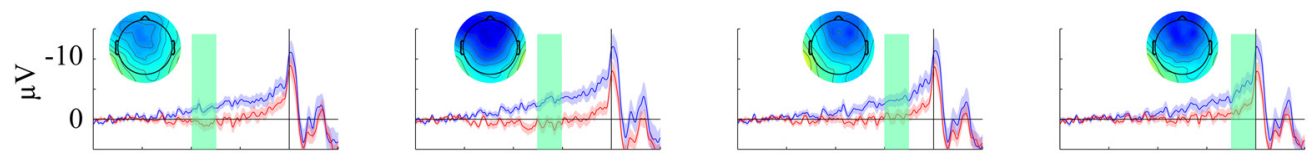

$\mathrm{FCz} / \mathrm{Cz}$
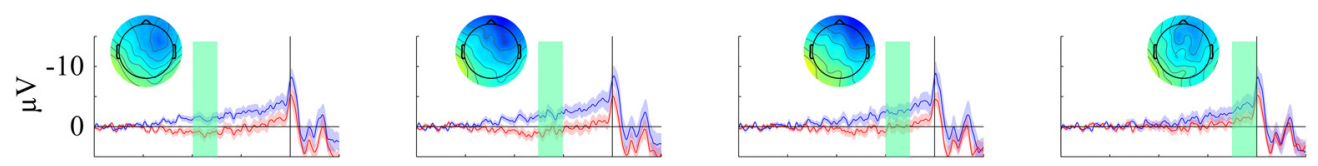

$\mathrm{FC} 4 / \mathrm{C} 4$
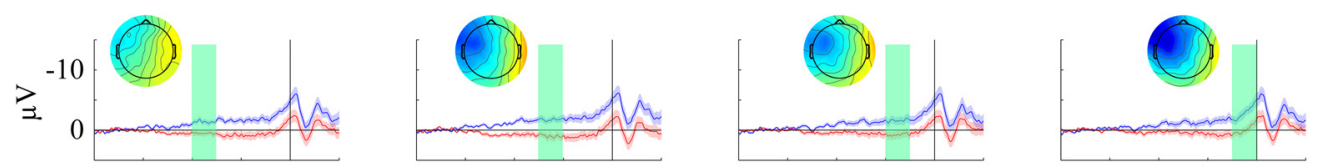

LRP
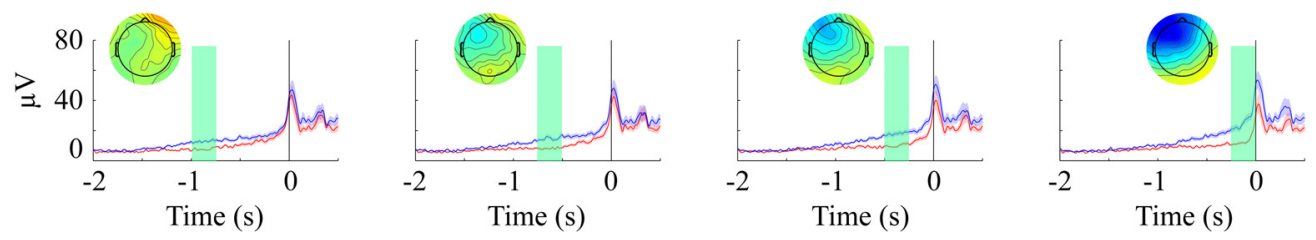

\section{GFP}

Low single-trial responses

High single-trial responses

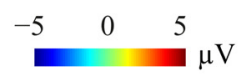

Figure 2. Comparison of ERPs for trials with high-versus low-amplitude responses estimated from the single-trial analysis. We divided trials into two groups according to the median split of the single-trial measures. We then recalculated the average ERP for trials of low-amplitude (red) versus high-amplitude (blue) estimates. The results in rows depict each of the RPs (left, center, and right channels), LRPs, and GFPs, and columns represent each of the four time windows from -1000 to 0 ms before movement. The time interval of each window over which the single-trial measures were estimated (for dividing high vs low responses) is highlighted by the shaded green area. We also plot the topographic map as the difference between the high- and low-response trials, computed within the highlighted time window for each ERP plot.

A

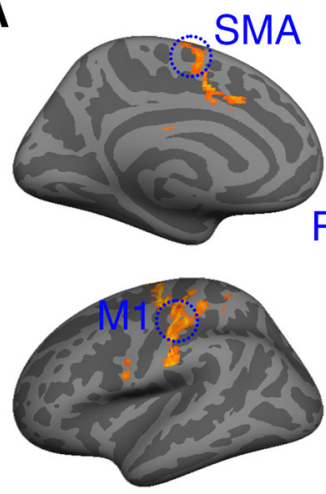

left

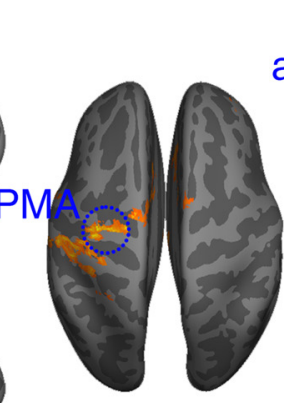

610

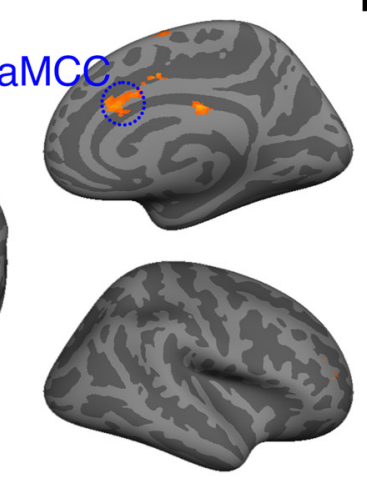

right
B

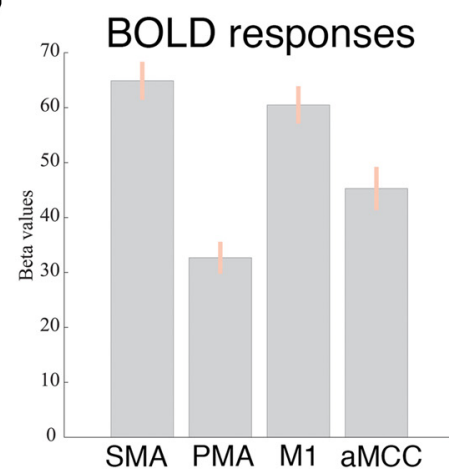

Figure 3. Whole-brain fMRl analysis. A, Significant activations are displayed on an inflated brain surface in which dark gray indicates the gyri and light gray indicates the sulci. Locations of aMCC, SMA, PMA, and $\mathrm{M} 1$ activation peaks are highlighted by the blue circles. Significant activations are identified by a cluster-level threshold $p_{\mathrm{FWE}}<0.05$, with clusters identified by a height threshold $p_{\text {uncorrected }}<0.00005$. $\boldsymbol{B}$, Mean parameter estimates of these ROIs are depicted, showing a similar level of activation responses in SMA and M1, both stronger than the activation responses in the PMA and aMCC.

high are associated with significantly greater BOLD activation in the aMCC.

\section{Effective connectivity}

We constructed two separate model spaces, first examining interactions specifically between SMA and aMCC, and subsequently adding primary motor cortex (M1) to examine its interactions with SMA and aMCC. The first model space was based on interactions only between aMCC and SMA, due to the effects found in our data above and the known crucial role of these regions in motor preparation processes (Fig. 5). The purpose of these models was to explain activation of both these regions during volun- 


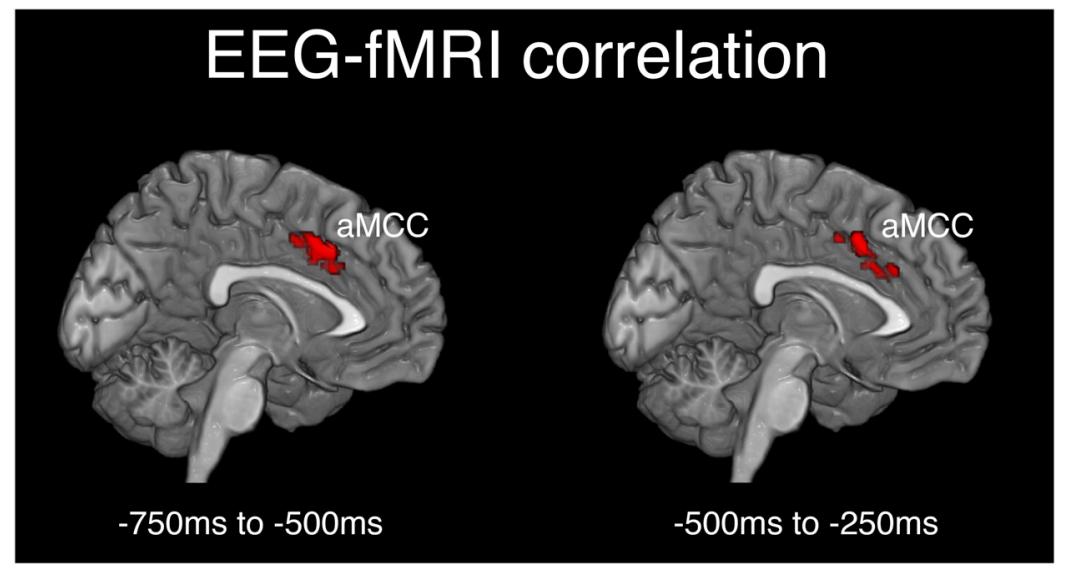

Figure 4. Areas of significant correlation between $\mathrm{AMRI}$ and EEG responses from the EEG-informed fMRI analysis. BOLD responses of the aMCC showed significant positive correlations with the trial-to-trial variation of the GFP in two time windows: -750 to $-500 \mathrm{~ms}$ and -500 to -250 ms before movement onset. Trials in which premovement GFP activity was larger were therefore associated with greater BOLD responses in this region of aMCC. Significant voxels were identified by a whole-brain voxelwise analysis using a corrected cluster-level threshold $p_{\mathrm{FWE}}<0.05$, with clusters defined by a height threshold $p_{\text {uncorrected }}<$ 0.001 .

Specification of GFP-derived modulators
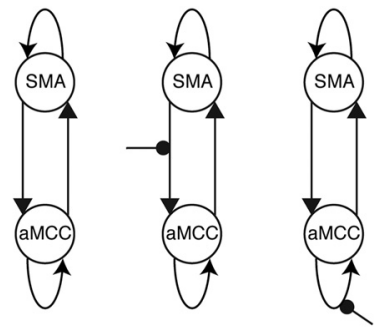

Interactions between SMA and aMCC
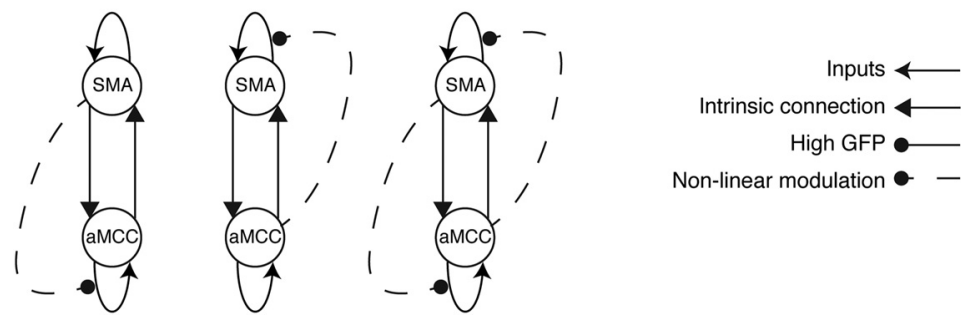

Figure 5. Construction of 36 model candidates. These models were constructed from a combination of three features: (1) the specification of GFP-derived modulators, (2) interactions between the SMA and aMCC, and (3) the specification of movement events as driving inputs.

tary movement as well as the positive modulation of BOLD responses in aMCC associated with large premovement GFP amplitudes. Hence, these models were motivated to encompass three different features: (1) the specification of contextual modulators derived from GFP, (2) interactions between aMCC and SMA, and (3) the specification of driving inputs representing the movement events. The first feature, using the GFP-derived contextual modulator, was introduced to explain the cause of the correlation between premovement GFP activity and BOLD responses in aMCC. Specifically, we tested whether this correlation in aMCC was better explained by the GFP (high or low) modulating the SMA to aMCC connection, or modulating the selffeedback connection of aMCC, or whether there was no influence of the GFP on the connections between these brain areas. The latter models (with no GFP modulatory effects) comprised fMRIonly DCM models and were included to examine the benefit of using GFP-derived parameters from the concurrently measured EEG data to model effective connectivity. The second feature tested the nature of the connectivity between SMA and aMCC and whether the modulations of these were better represented using bilinear or nonlinear models (Stephan et al., 2008). Nonlinear models comprised the activity of SMA or aMCC modulating the self-feedback connection of the other region (aMCC or SMA), or reciprocal interactions in which the activity of SMA and aMCC modulate the selffeedback connections of each other. The third feature tested whether the driving inputs acted directly on the activity of either aMCC or SMA alone, or on both regions.

Since activity in SMA already begins a few seconds before movement onset (Windischberger et al., 2008), it was not clear a priori whether the driving inputs of our DCMs should be modeled at movement onset or earlier to capture this premovement activity. We tested this idea by examining the driving inputs with different onsets to model the premovement activity. Here, we shifted the event onsets by $1 \mathrm{~s}$ or $2 \mathrm{~s}$ earlier and compared these models with those having the driving inputs representing the time of movement onset. The results showed that the models without shifting the time of the movement events performed significantly better than those with the shifted onsets. Hence, we selected the onsets of the movement events constituting the driving inputs for our following DCMs. In total, we included 36 models in the model space, with each model representing a unique combination of the three features allowing us to examine specific hypotheses on the interactions between aMCC and SMA and the influence of GFP on those interactions (Fig. 5).

Model inversion and selection strongly favored a single best-fitting model from our model space (Fig. 6). Within the best-fitting model, the positive correlation between the GFP and activity of aMCC was best explained by positive modulation of the connection from SMA to aMCC when GFP activity was large. A nonlinear and reciprocal modulation was found between the activity of aMCC and SMA, with the self-feedback connection of SMA regulated by aMCC, and the self-feedback connection of aMCC regulated by SMA. This model also featured driving inputs to both aMCC and SMA. We also performed familywise model selection, in which models with shared features were grouped together in the same family, to examine the optimal model features. We found that the individual features characterizing the best-fitting model indeed were optimal at the family-level selection when considered separately. For example, the feature that the GFP contextual modulator regulated the SMA-aMCC connection was more favorable than those including the modulation on the self-feedback connection 
A

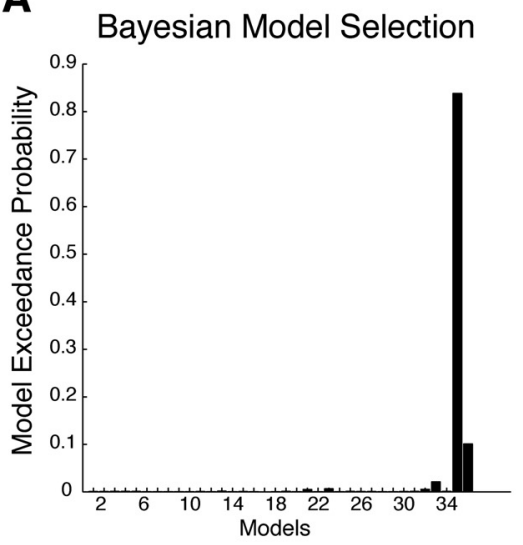

\section{C}

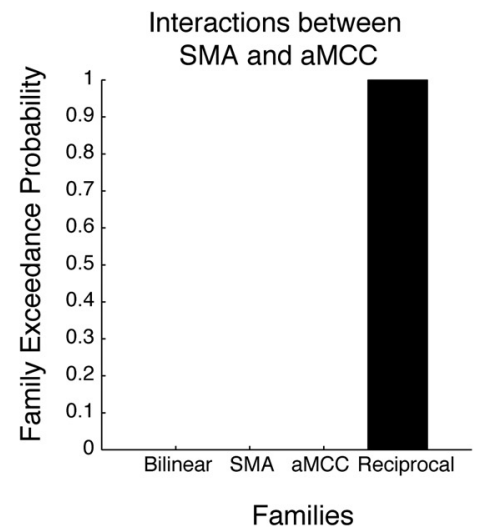

B

Intrinsic Connectivity

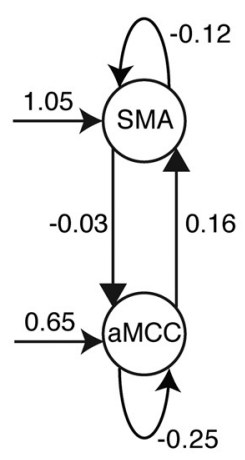

Specification of

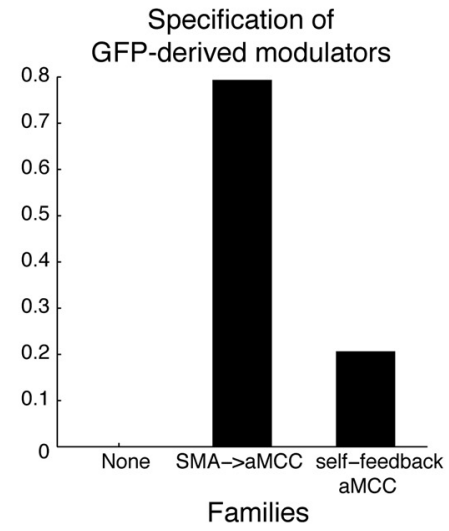

Modulation Effects
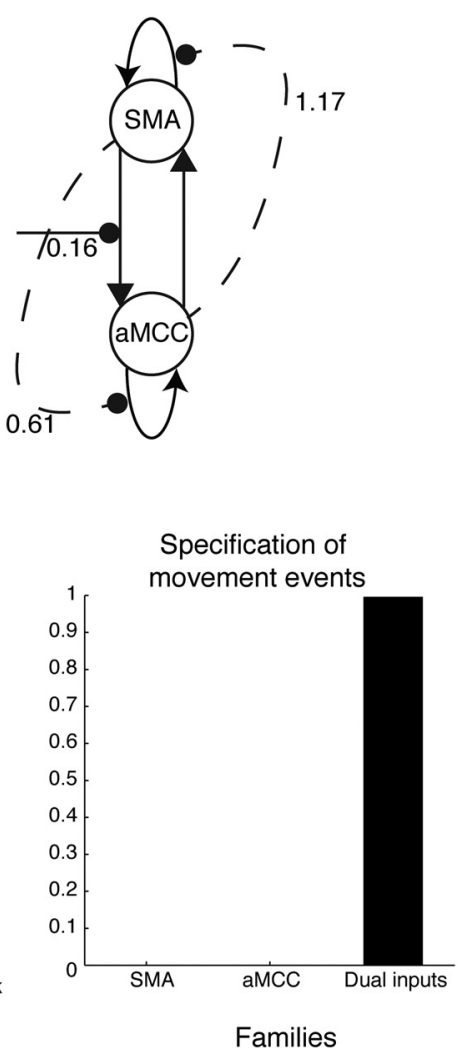

Figure 6. Model selection. Results of the BMS analysis identifying the best-fitting model among all 36 competing candidates $(\boldsymbol{A})$ and the connection strengths of the best-fitting model separated by intrinsic connections and modulation effects $(\boldsymbol{B})$. $\boldsymbol{C}$, Results of familywise model selection based on the three features tested across the model space. The best-fitting model comprised each of the features that were identified as optimal in the familywise model selection.

of aMCC, or no regulation of the GFP. These results reassuringly confirmed that the best-fitting model consisted of an optimal combination of all favorable features.

Based on the optimal model found in the first model space, we additionally examined a second model space: this was based on the winning two-region (aMCC and SMA) model plus the additional inclusion of M1. We constructed six models testing the interactions between $\mathrm{M} 1$ and these other two regions (Fig. 7A). These models tested whether M1 receives only forward connections from aMCC or SMA, or both, or whether M1 has bidirectional connections with aMCC or SMA, or both. Results of the BMS analysis revealed that the model having the bidirectional connections between M1 and both aMCC and SMA was significantly more favorable than its counterparts (Fig. $7 B, C$ ).

\section{Discussion}

Our study used model-driven integration of simultaneous EEG and fMRI data to examine the role of the aMCC and SMA in the preparation for voluntary action. We showed that premovement neural activity reflected in the GFP is strongly associated with $\mathrm{BOLD}$ responses in the aMCC. This result highlights the aMCC as a crucial region contributing to the sustained activity of the RP before movement. Furthermore, our DCM analyses suggest that the aMCC and SMA comodulate each other to maintain sustained activity over the premovement period, and ultimately to trigger movement via activity in the primary motor cortex. We speculate that connection patterns of the SMA and aMCC hold signatures of their functional role in the preparation of selfgenerated action.

\section{Correlation between GFP and the aMCC activity}

Despite the different temporal resolution of EEG compared with fMRI and their different neuronal determinants, our analysis revealed a positive correlation between premovement GFP activity and BOLD signal in the aMCC. Crucially, this association was observed during the time window between 700 and $400 \mathrm{~ms}$ before movement. This period is in concordance with the early component of the RP, commonly identified between 1500 and $500 \mathrm{~ms}$ preceding movement (Shibasaki and Hallett, 2006). Importantly, the early RP component occurs before lateralization of activity to the hemisphere contralateral to movement at $\sim 400 \mathrm{~ms}$ (Shibasaki and Hallett, 2006). Previous studies using source analysis have also localized the early RP component to the cingulate cortex (Praamstra et al., 1996; Ball et al., 1999; Toma et al., 2002). While it must be noted that the correlations reported in our study were based on GFP, rather than specifically RP or LRP directly, this is due to the vastly superior signal-to-noise ratio of GFP compared with single-channel amplitudes (e.g., for RP and LRP) when estimating single-trial activity for the EEG-informed fMRI analysis. GFP considers signals from all channels across the scalp as a measure of overall field strength (Lehmann and Skrandies, 1980). Because the RP is known to have a widespread distribution across the scalp and stable topography over time (Shibasaki and Hallett, 2006), GFP provides an ideal measure of neural activity of 


\section{A Model space}

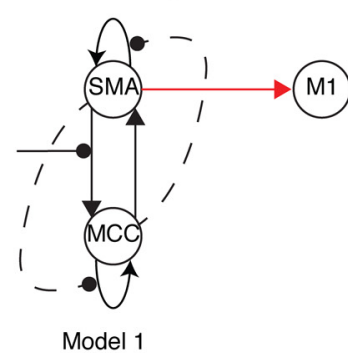

Model 1
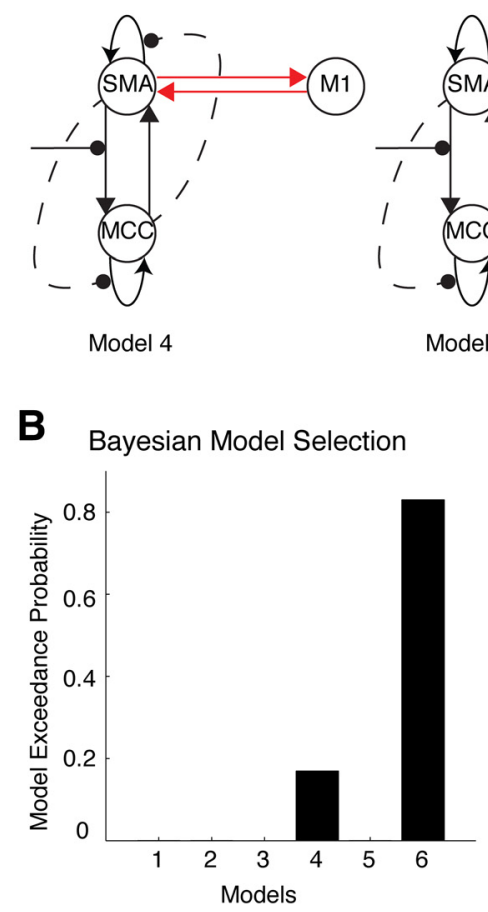

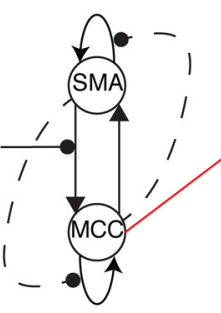

Model 2

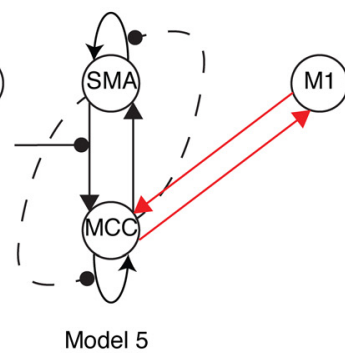

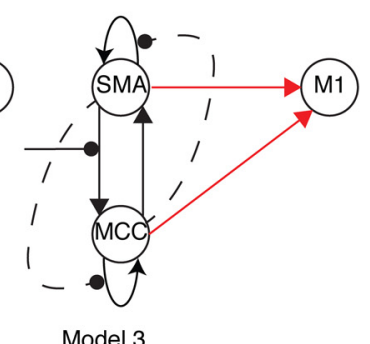

Model 3

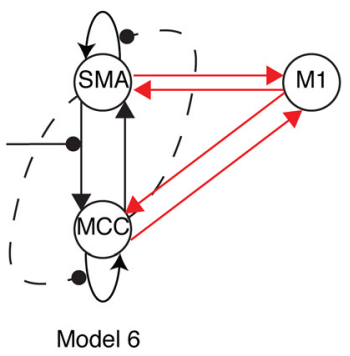

C Connection strengths of the best fitting model

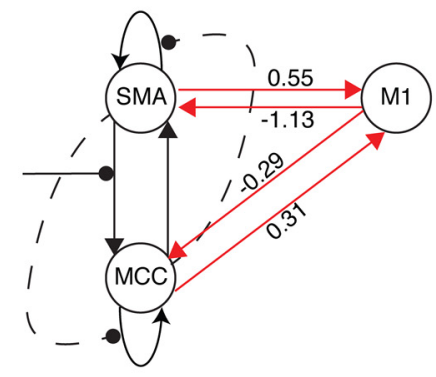

Figure 7. Interactions between M1 and aMCC and SMA based on the optimal model shown in Figure 6. $\boldsymbol{A}$, The model space comprised six models examining whether aMCC and SMA provide feedforward or bidirectional connections to M1. $\boldsymbol{B}$, The BMS analysis showed a clear best-fitting model from among the six models. $C$, The best-fitting model comprised of bidirectional connections between $\mathrm{M} 1$ and both SMA and aMCC.

the RP for single-trial analysis. Our results firmly suggest that the aMCC contributes to premovement cortical activity reflected in the early RP component.

We do not suggest that the aMCC is the sole generator of premovement activity, but rather that it facilitates the large-scale distributed cortical activity, reflected by GFP, during the preparation of self-paced action. The aMCC is positioned as a topographical hub of the rich club, connecting with a wide network of brain regions (van den Heuvel and Sporns, 2011). Its functionality is thought to be crucial to a broad range of cognitive processes such as reward-dependent decision making (Mulert et al., 2008), error-related processing (Debener et al., 2005), and rewardrelated actions (Shima and Tanji, 1998). In the present context of voluntary movements, the aMCC is thought to be involved in the process of action selection (Matsumoto et al., 2003; Lau et al., 2004; Rushworth et al., 2004; Cunnington et al., 2006) as well as action initiation (Hoffstaedter et al., 2013). Our results advance the crucial involvement of the aMCC in self-generated action by showing an important contribution of the aMCC to the overall increase in cortical activity before movement evident in GFP. That is, the aMCC is a crucial modulator or driver of neural activity across the cortex in preparation for action. However, our

results do not preclude other brain regions such as the SMA contributing to neural sources of the early RP component (Praamstra et al., 1996; Ball et al., 1999).

\section{Effective connectivity}

Our DCM results suggest that the aMCC and SMA are linked in a reciprocal network and act to sustain each other's activity. Crucially, the strength of their interaction is associated with the level of premovement cortical activity of the early RP as reflected in the GFP. We were able to identify a clearly superior model among all 36 plausible networks that also combined each of the features found to be most favorable in familywise model selection (Fig. 6). Our key finding is that both the aMCC and SMA modulate each other's self-feedback connections via nonlinear interactions. In DCM, the self-feedback connections determine the self-inhibition of activity in each region (Friston et al., 2003). These reciprocal interactions by which the aMCC and SMA modulate each other's self-feedback connections therefore allow these regions to maintain sustained activity in the absence of other driving input as a self-exciting system. Overall, our findings suggest that the SMA modulates the activity of the aMCC in accordance with premovement neural activity as reflected in the GFP. The aMCC, in turn, modulates activity of the SMA and its selffeedback connection to sustain the activity of this network. Hence, the aMCC and SMA mutually interact to maintain neural activity, rather than relying upon one region to dominate network activity during the premovement period. Here, our study provides a computational account of the notion that the aMCC and SMA interact to sustain their activity over time in preparation for self-generated action, and the strength of their interaction is associated with the amplitude of cortical activity over the early RP.

One criticism of our interpretation could be that our DCMs integrate neural activity of both the preparation and execution phases of action, which were driven by inputs specified at the moment of movement onset. In other words, we cannot disambiguate the premovement from the postmovement stage of action due to the slow timescales of the HRF. Our model benefits from the timing information specific to the influence of the GFP during the planning stage of action (Fig. 4). We thus argue that the modulation of GFP ties effective connectivity interactions of the aMCC and SMA to particular time points that are specific to the preparation stage. Hence, our results advance upon previous findings showing the involvement of the aMCC and SMA in the planning of internally generated action (Cunnington et al., 2002, 2005) by suggesting that the self-exciting system of the aMCC and SMA is essential to sustain premovement activity and influence the transition from preparation of action to movement initiation. Furthermore, when we included the primary motor cortex (M1) in our model space, the most favorable model was 
characterized by a positive influence of the medial aMCC and SMA regions on the M1 contralateral to movement. This is consistent with previous studies that have examined motor network activity with DCM, showing that the SMA in particular enhances coupling with the M1 contralateral to movement to drive movement initiation (Grefkes et al., 2008; Kasess et al., 2008; Pool et al., 2013) and suppresses M1 activity for actions not to be performed, i.e., in the case of imagined movements (Kasess et al., 2008) or in the ipsilateral M1 for unimanual movements (Grefkes et al., 2008). Overall, these results point to a particular role for the medial regions, aMCC and SMA, maintaining premovement activity in readiness for action and enhancing coupling with the M1 contralateral to movement for movement execution.

There are unanswered questions that arise from the present study. Premovement neural activity is greatest over the late components of the RP immediately before movement; however, we found no BOLD correlations with either RP or LRP amplitudes or GFP activity over this period. We speculate that brain activity reflected in the late component is more likely to encode the execution stage of action (Shibasaki and Hallett, 2006), hence accounting for the lack of a robust correlation with the aMCC and SMA, since these regions are more closely involved in the planning stage. Another question concerns the association between activity of the aMCC and the RP, as well as SMA-aMCC interactions, for externally cued actions rather than self-paced actions. Previous research has shown that the SMA and aMCC are more active and the magnitude of the early RP component is greater for voluntary self-paced movement than for externally cued actions (Cunnington et al., 1995, 2002; Jahanshahi et al., 1995). Further research is needed to determine whether the self-sustaining SMA-aMCC network that we find here is involved only in maintaining the long period of sustained premovement activity before self-paced actions, or whether this system is engaged more generally in all movement initiation.

We also acknowledge that the driving inputs specified at the onset of movement provided the most likely models of our data; however, the underlying mechanisms that in turn drive the activity in the aMCC and SMA are not yet explicated. This is an interesting question since it speaks directly to a fundamental issue in human volition, namely which regions or what mechanisms initiate voluntary movements (Haggard, 2008; Roskies, 2010). The pattern of nonlinear connectivity in a self-regulating pair of regions represents an ideal substrate for the production of subtle dynamical instabilities in models of large-scale neural systems (Breakspear, 2002, 2003). It is possible that the premovement modulation corresponds to the production of weak instabilities preceding movement, allowing action to be triggered in midline motor regions, similar to the "ignition" of perceptual processes in frontoparietal regions by dynamical instabilities (Friston et al., 2012). The final trigger might arise via an external input to the region from an area such as the basal ganglia (Brotchie et al., 1991; Cunnington et al., 1997), or by fluctuations within the motor system itself, meaning that the final step in volitional action arises in a system primed to be receptive to a small (and possibly random) push. Accordingly, the slow changes in largescale potentials may correspond to the "critical slowing" of neural dynamics that precede such instabilities (Kelso, 2010; Aburn et al., 2012). We suggest that future studies could build on our findings through dynamical analyses of the fast oscillations in our data (Freyer et al., 2012), or by extending the structural model further by building a more complete network underpinning the preparation for action.

\section{References}

Aburn MJ, Holmes CA, Roberts JA, Boonstra TW, Breakspear M (2012) Critical fluctuations in cortical models near instability. Front Physiol 3:331. CrossRef Medline

Allen PJ, Josephs O, Turner R (2000) A method for removing imaging artifact from continuous EEG recorded during functional MRI. Neuroimage 12:230-239. CrossRef Medline

Ball T, Schreiber A, Feige B, Wagner M, Lücking CH, Kristeva-Feige R (1999) The role of higher-order motor areas in voluntary movement as revealed by high-resolution EEG and fMRI. Neuroimage 10:682-694. CrossRef Medline

Breakspear M (2002) Nonlinear phase desynchronization in human electroencephalographic data. Hum Brain Mapp 15:175-198. CrossRef Medline

Breakspear M, Terry JR, Friston KJ (2003) Modulation of excitatory synaptic coupling facilitates synchronization and complex dynamics in a biophysical model of neuronal dynamics. Network 14:703-732. CrossRef Medline

Brotchie P, Iansek R, Horne MK (1991) Motor function of the monkey globus pallidus. 2. Cognitive aspects of movement and phasic neuronal activity. Brain 114:1685-1702. CrossRef Medline

Cerutti S, Baselli G, Liberati D, Pavesi G (1987) Single sweep analysis of visual evoked potentials through a model of parametric identification. Biol Cybern 56:111-120. CrossRef Medline

Cunnington R, Iansek R, Bradshaw JL, Phillips JG (1995) Movementrelated potentials in Parkinson's disease. Presence and predictability of temporal and spatial cues. Brain 118:935-950. CrossRef Medline

Cunnington R, Iansek R, Johnson KA, Bradshaw JL (1997) Movementrelated potentials in Parkinson's disease. Motor imagery and movement preparation. Brain 120:1339-1353. CrossRef Medline

Cunnington R, Windischberger C, Deecke L, Moser E (2002) The preparation and execution of self-initiated and externally-triggered movement: a study of event-related fMRI. Neuroimage 15:373-385. CrossRef Medline

Cunnington R, Windischberger C, Deecke L, Moser E (2003) The preparation and readiness for voluntary movement: a high-field event-related fMRI study of the Bereitschafts-BOLD response. Neuroimage 20:404412. CrossRef Medline

Cunnington R, Windischberger C, Moser E (2005) Premovement activity of the presupplementary motor area and the readiness for action: studies of time-resolved event-related functional MRI. Hum Mov Sci 24:644-656. CrossRef Medline

Cunnington R, Windischberger C, Robinson S, Moser E (2006) The selection of intended actions and the observation of others' actions: a timeresolved fMRI study. Neuroimage 29:1294-1302. CrossRef Medline

Debener S, Ullsperger M, Siegel M, Fiehler K, von Cramon DY, Engel AK (2005) Trial-by-trial coupling of concurrent electroencephalogram and functional magnetic resonance imaging identifies the dynamics of performance monitoring. J Neurosci 25:11730-11737. CrossRef Medline

Delorme A, Makeig S (2004) EEGLAB: an open source toolbox for analysis of single-trial EEG dynamics including independent component analysis. J Neurosci Methods 134:9-21. CrossRef Medline

Duncan NW, Northoff G (2013) Overview of potential procedural and participant-related confounds for neuroimaging of the resting state. J Psychiatry Neurosci 38:84-96. CrossRef Medline

Eichele T, Specht K, Moosmann M, Jongsma ML, Quiroga RQ, Nordby H, Hugdahl K (2005) Assessing the spatiotemporal evolution of neuronal activation with single-trial event-related potentials and functional MRI. Proc Natl Acad Sci U S A 102:17798-17803. CrossRef Medline

Eickhoff SB, Stephan KE, Mohlberg H, Grefkes C, Fink GR, Amunts K, Zilles K (2005) A new SPM toolbox for combining probabilistic cytoarchitectonic maps and functional imaging data. Neuroimage 25:1325-1335. CrossRef Medline

Erdler M, Beisteiner R, Mayer D, Kaindl T, Edward V, Windischberger C, Lindinger G, Deecke L (2000) Supplementary motor area activation preceding voluntary movement is detectable with a whole-scalp magnetoencephalography system. Neuroimage 11:697-707. CrossRef Medline

Freyer F, Roberts JA, Ritter P, Breakspear M (2012) A canonical model of multistability and scale-invariance in biological systems. PLoS Comput Biol 8:e1002634. CrossRef Medline

Friston K (2010) The free-energy principle: a unified brain theory? Nat Rev Neurosci 11:127-138. CrossRef Medline 
Friston KJ, Harrison L, Penny W (2003) Dynamic causal modelling. Neuroimage 19:1273-1302. CrossRef Medline

Friston K, Breakspear M, Deco G (2012) Perception and self-organized instability. Front Comput Neurosci 6:44. CrossRef Medline

Grefkes C, Eickhoff SB, Nowak DA, Dafotakis M, Fink GR (2008) Dynamic intra- and interhemispheric interactions during unilateral and bilateral hand movements assessed with fMRI and DCM. Neuroimage 41:13821394. CrossRef Medline

Haggard P (2008) Human volition: towards a neuroscience of will. Nat Rev Neurosci 9:934-946. CrossRef Medline

Hoffstaedter F, Grefkes C, Zilles K, Eickhoff SB (2013) The "what" and "when" of self-initiated movements. Cereb Cortex 23:520-530. CrossRef Medline

Hoffstaedter F, Grefkes C, Caspers S, Roski C, Palomero-Gallagher N, Laird AR, Fox PT, Eickhoff SB (2014) The role of anterior midcingulate cortex in cognitive motor control: evidence from functional connectivity analyses. Hum Brain Mapp 35:2741-2753. CrossRef Medline

Jahanshahi M, Jenkins IH, Brown RG, Marsden CD, Passingham RE, Brooks DJ (1995) Self-initiated versus externally triggered movements. I. An investigation using measurement of regional cerebral blood flow with PET and movement-related potentials in normal and Parkinson's disease subjects. Brain 118:913-933. CrossRef Medline

Jansen M, White TP, Mullinger KJ, Liddle EB, Gowland PA, Francis ST, Bowtell R, Liddle PF (2012) Motion-related artefacts in EEG predict neuronally plausible patterns of activation in fMRI data. Neuroimage 59:261-270. CrossRef Medline

Kasess CH, Windischberger C, Cunnington R, Lanzenberger R, Pezawas L, Moser E (2008) The suppressive influence of SMA on M1 in motor imagery revealed by $\mathrm{fMRI}$ and dynamic causal modeling. Neuroimage 40 : 828-837. CrossRef Medline

Kelso JA (2010) Instabilities and phase transitions in human brain and behavior. Front Hum Neurosci 4:23. CrossRef Medline

Kornhuber HH, Deecke L (1964) Hirnpotentialanderungen beim Menschen vor und nach Willkurbewegungen, dargestellt mit Magnetbandspeicherung und Ruckwartsanalyse. Pflugers Arch 281:52.

Lang W, Cheyne D, Kristeva R, Beisteiner R, Lindinger G, Deecke L (1991) Three-dimensional localization of SMA activity preceding voluntary movement. A study of electric and magnetic fields in a patient with infarction of the right supplementary motor area. Exp Brain Res 87:688695. CrossRef Medline

Lau HC, Rogers RD, Ramnani N, Passingham RE (2004) Willed action and attention to the selection of action. Neuroimage 21:1407-1415. CrossRef Medline

Lehmann D, Skrandies W (1980) Reference-free identification of components of checkerboard-evoked multichannel potential fields. Electroencephalogr Clin Neurophysiol 48:609-621. CrossRef Medline

Logothetis NK, Pauls J, Augath M, Trinath T, Oeltermann A (2001) Neurophysiological investigation of the basis of the fMRI signal. Nature 412: 150-157. CrossRef Medline

Matsumoto K, Suzuki W, Tanaka K (2003) Neuronal correlates of goalbased motor selection in the prefrontal cortex. Science 301:229-232. CrossRef Medline

Moosmann M, Schönfelder VH, Specht K, Scheeringa R, Nordby H, Hugdahl K (2009) Realignment parameter-informed artefact correction for simultaneous EEG-fMRI recordings. Neuroimage 45:1144-1150. CrossRef Medline

Mulert C, Seifert C, Leicht G, Kirsch V, Ertl M, Karch S, Moosmann M, Lutz J, Möller H-J, Hegerl U, Pogarell O, Jäger L (2008) Single-trial coupling of EEG and fMRI reveals the involvement of early anterior cingulate cortex activation in effortful decision making. Neuroimage 42:158-168. CrossRef Medline

Nguyen VT, Cunnington R (2014) The superior temporal sulcus and the
N170 during face processing: single trial analysis of concurrent EEGfMRI. Neuroimage 86:492-502. CrossRef Medline

Nguyen VT, Breakspear M, Cunnington R (2013) Fusing concurrent EEGfMRI with dynamic causal modeling: application to effective connectivity during face perception. Neuroimage S1053-8119(13)00730-1. CrossRef

Niazy RK, Beckmann CF, Iannetti GD, Brady JM, Smith SM (2005) Removal of FMRI environment artifacts from EEG data using optimal basis sets. Neuroimage 28:720-737. CrossRef Medline

Paus T (2001) Primate anterior cingulate cortex: where motor control, drive and cognition interface. Nat Rev Neurosci 2:417-424. CrossRef Medline

Paus T, Petrides M, Evans AC, Meyer E (1993) Role of the human anterior cingulate cortex in the control of oculomotor, manual, and speech responses: a positron emission tomography study. J Neurophysiol 70:453469. Medline

Penny WD (2012) Comparing dynamic causal models using AIC, BIC and free energy. Neuroimage 59:319-330. CrossRef Medline

Picard N, Strick PL (1996) Motor areas of the medial wall: a review of their location and functional activation. Cereb Cortex 6:342-353. CrossRef Medline

Pool EM, Rehme AK, Fink GR, Eickhoff SB, Grefkes C (2013) Network dynamics engaged in the modulation of motor behavior in healthy subjects. Neuroimage 82:68-76. CrossRef Medline

Praamstra P, Stegeman DF, Horstink MW, Cools AR (1996) Dipole source analysis suggests selective modulation of the supplementary motor area contribution to the readiness potential. Electroencephalogr Clin Neurophysiol 98:468-477. CrossRef Medline

Roskies AL (2010) How does neuroscience affect our conception of volition? Annu Rev Neurosci 33:109-130. CrossRef Medline

Rushworth MF, Walton M, Kennerley SW, Bannerman DM (2004) Action sets and decisions in the medial frontal cortex. Trends Cogn Sci 8:410417. CrossRef Medline

Shibasaki H, Hallett M (2006) What is the Bereitschaftspotential? Clin Neurophysiol 117:2341-2356. CrossRef Medline

Shima K, Tanji J (1998) Role for cingulate motor area cells in voluntary movement selection based on reward. Science 282:1335-1338. CrossRef Medline

Stephan KE, Marshall JC, Penny WD, Friston KJ, Fink GR (2007) Interhemispheric integration of visual processing during task-driven lateralization. J Neurosci 27:3512-3522. CrossRef Medline

Stephan KE, Kasper L, Harrison LM, Daunizeau J, den Ouden HE, Breakspear M, Friston KJ (2008) Nonlinear dynamic causal models for fMRI. Neuroimage 42:649-662. CrossRef Medline

Toma K, Matsuoka T, Immisch I, Mima T, Waldvogel D, Koshy B, Hanakawa T, Shill H, Hallett M (2002) Generators of movement-related cortical potentials: fMRI-constrained EEG dipole source analysis. Neuroimage 17:161-173. CrossRef Medline

Tzourio-Mazoyer N, Landeau B, Papathanassiou D, Crivello F, Etard O, Delcroix N, Mazoyer B, Joliot M (2002) Automated anatomical labeling of activations in SPM using a macroscopic anatomical parcellation of the MNI MRI single-subject brain. Neuroimage 15:273-289. CrossRef Medline

van den Heuvel MP, Sporns O (2011) Rich-club organization of the human connectome. J Neurosci 31:15775-15786. CrossRef Medline

Windischberger C, Cunnington R, Lamm C, Lanzenberger R, Langenberger H, Deecke L, Bauer H, Moser E (2008) Time-resolved analysis of fMRI signal changes using brain activation movies. J Neurosci Methods 169: 222-230. CrossRef Medline

Yazawa S, Ikeda A, Kunieda T, Ohara S, Mima T, Nagamine T, Taki W, Kimura J, Hori T, Shibasaki H (2000) Human presupplementary motor area is active before voluntary movement: subdural recording of Bereitschaftspotential from medial frontal cortex. Exp Brain Res 131:165-177. CrossRef Medline 